\title{
Die Bewertung von Risiken durch Naturgefahren mit spezieller Berïcksichtigung der Risikoaversion
}

\section{Thomas Plattner, Zürich}

\section{Der risiko-orientierte Umgang mit Naturgefahren}

Bei Risiken aus Naturgefahren müssen aus technischen und ökonomischen Gründen gewisse Restrisiken akzeptiert werden. Um das Ausmass dieser Risiken abschätzen zu können, ist die Beantwortung folgender Fragen wichtig (Abb. 1: HolleNSTEIN 1997: siehe auch den Beitrag von H. KiEnHolz in diesem Heft):

- Was kann passieren? - Risikoanalyse

- Was darf passieren? - Risikobewertung

Die Risikoanalyse ist ein natur- und ingenieurwissenschaftlicher Ansatz, der das Risiko in quantitativer Art und Weise charakterisiert (SOCIETY OF RISK ANALYSIS 2004) und als objektiv messbaren Schadenerwartungswert $R_{o b j}$ definiert (und somit die Frage beantwortet «was kann passieren?» (Hollenstein 1997)). Es existieren prozessspezifische Modelle, deren Anwendung detaillierte Informationen über das Ausmass und die Eintretenswahrscheinlichkeit eines Schadens liefern. Eine umfassende Risikoanalyse beinhaltet die Gefahrenidentifikation, die Ereignisabschätzung, die Bestimmung von Schadenobjekten und die Abschätzung der Ereignisauswirkungen. Das objektive Risiko $R_{a b l}$ wird in seiner einfachsten Form als Schadenerwartungswert (Funktion der Eintretenswahrscheinlichkeit eines Ereignisses $F$ und dem Ausmass, der mit diesem Ereignis assoziierten Konsequenzen $E$ ) berechnet:

$$
R_{o b, j}=F \bullet E
$$

Die Risikobewertung ist ein sozio-politischer und ethisch-moralischer Prozess. Mittels dem akzeptablen Risiko $R_{\text {acc }}$ wird die Frage "was darf passieren?" beantwortet und damit ein Urteil über die Akzeptabilität von Risiken zugelassen (Society of RISK ANALysis 2004). Im Rahmen des Risikomanagements erfolgt, auf der Basis des Vergleichs des objektiven Risikos mit dem akzeptablen Risiko, die Planung der notwendigen Massnahmen. Folgende Szenarien sind denkbar (vgl. Abb. 1):

$$
\begin{array}{ll}
\text { Szenario } & \text { Folge } \\
R_{o b j}>R_{a c c} & \text { Reduktion von } R_{o b j} \text { oder Erhöhung } \\
& \text { von } R_{a c c} \\
R_{o b b j}=R_{a c c} & \text { Reduktion/Stabilisierung von } R_{o b j} \\
R_{o b j}<R_{a c c} & \text { oder Erhöhung von } R_{a c c} \\
\text { keine Massnahmen }
\end{array}
$$

Unter Berücksichtigung ökonomischer und technischer Prinzipien (Heinimann 2002) sind jene Risiken, die inakzeptabel sind, zu reduzieren und die anderen Risiken daran zu hindern, inakzeptabel zu werden.

Bislang besteht ein Mangel an Methoden. um die gesellschaftliche Risikobewertung zu simulieren. Solche Modelle sind aber hilfreich. da mit geringem finanziellen und zeitlichen Aufwand wichtige Informationen zur Risikoakzeptanz erhältlich sind.

\section{Risikowahrnehmung und Risikobewertung}

\subsection{Relevante Faktoren der Risikowahrnehmung}

Eine Vielzahl von Faktoren beeinflusst die Risikowahrnehmung. Psychologische Strategien (Heuristiken) helfen, mental mit der Unsicherheit bei Entscheidungen umzugehen und komplexe Fragestellungen zu vereinfachen (Tversky \& KahNeman 1974). DougLAS \& Wildavsky (1982) sowie Wildavsky \& Dake (1990) weisen nach, dass der sozio-kulturelle Hintergrund einer Person Einfluss auf die Wahrnehmung und Bewertung von Risiken hat. Diese wird zudem durch soziale Interaktion beeinflusst. Daraus resultiert meist eine verstärkte Risikowahrnehmung (KaSPERson et al. 1988). STARr (1969) wies zudem darauf hin. dass freiwillig eingegangene Risiken bis zu tausend Mal grösser sein können als unfreiwillig eingegangene Risiken und dennoch akzeptabel sind. Die Bedeutung der Freiwilligkeit wurde auch von Fischroff et al. (1979) erläutert. Gemeinsam mit den Arbeiten von Stovic et al. (1981), Stovic et al. (1986) und Stovic (1987) weisen sie auch auf die Bedeutung des Faktors Wissen hin. Wissen ist dabei zu verstehen als das technisch-wissenschaftliche Wissen (risks known to science) oder das subjektiv-intuitive Wissen von Laien (risks known to those exposed). Die Furcht (dread) erklärt z.B. bei technischen Risiken (wie Nuklearenergie) einen Grossteil der Risikowahrnehmung. Zusammenfassend geht Hollenstein (1997) davon aus, dass die Bedrohlichkeit eines Ereignisses (Furcht) und die Vertrautheit mit einem Ereignis (subjektiv-intuitives Wissen und Erfahrung) wichtig sind. Zudem spielen die Freiwilligkeit, mit der das Risiko eingegangen wird und die Reduzierbarkeit des Risikos (technisch-wissenschaftliches Wissen) eine Rolle.

\subsection{Bestehende Ansätze der Risikobewertung}

Wichtige Ansätze zur Festlegung akzeptabler Risiken 


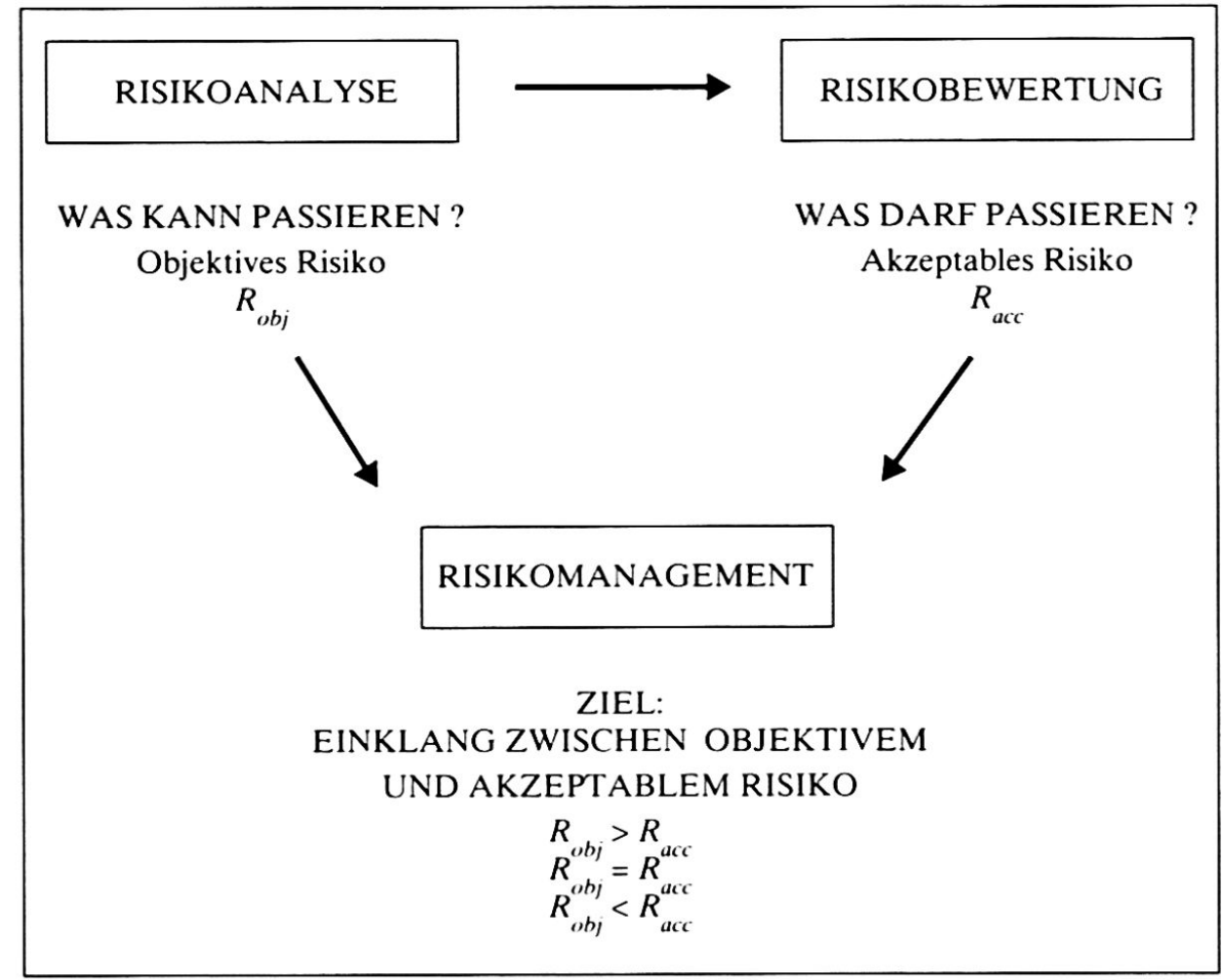

Abb. 1: Risiko-orientierter Umgang mit Naturgefahren

Risk-oriented approach to natural hazards

Une approche orientée vers le risque en matière de dangers naturels

Quelle: HoLLENSTEIN 1997 (verändert)

sind die Akzeptanzlinie (FARMER-Linie, boundary line) (Eidgenössisches Departement des InNeRn 1989; Farmer 1967) und der Aversions-Term (Bohnenblust \& Troxler 1987; BUNDESAMt Für UMWELT, WALD UND LANDSCHAFT 1999; SCHNEIDER 1985). Beide verwenden die Risikoaversion $\alpha$, die eine Funktion des direkten Schaden- und Konsequenzausmasses $E$ eines Ereignisses (z.B. Anzahl Tote oder Verletzte) darstellt

$$
\alpha=f(E)=\alpha(E)
$$

Durch $\alpha$ wird die öffentliche Beurteilung eines Risikos in die Risikoberechnung integriert. Daraus resultiert eine bewusste überproportionale Gewichtung von Ereignissen mit grossen Schäden, die auf folgenden Überlegungen beruht (Bohnenblust \& Schneider 1984):

- Fehleinschätzungen von Risiken: Risiken können im Bezug auf das Ausmass des Schadens und auf die Eintretenswahrscheinlichkeit eines Schadens falsch eingeschätzt werden.

- Community effect: wegen unterschiedlichen Regenerationsfähigkeiten besteht eine Differenz in der Wahrnehmung, wenn zehn Leute aus einer kleinen Gemeinschaft (Familie, Dorf) getötet werden, oder zehn Menschen, die in keinem Bezug zueinander stehen. Somit hängt die Risikoaversion davon ab, ob eine Gemeinschaft durch ein grosses Schadenereignis (= katastrophales Ereignis) in ihrer Existenz gefährdet ist (die Anzahl an Toten an sich ist nicht der ausschlaggebende Punkt).

- Signaleffekt: die Öffentlichkeit wird durch Ereignisse mit katastrophalen Schadensauswirkungen aufgeschreckt, da sie zuvor kaum Notiz von den Ereignissen mit wenigen (oder keinen) Todesopfern genommen hat. Katastrophale Ereignisse machen die Gefährdung bewusst und verursachen daher überproportionale Reaktionen (die bei gleicher Opferanzahl bei mehreren kleinen Ereignissen nicht auftreten würden).

Die Bewertungsansätze unterscheiden sich bei der Integration der Risikoaversion. Die Akzeptanzlinie ist eine Linie konstanten Risikos auf einem doppeltlogarithmischen Kurvendiagramm mit der Eintretenswahrscheinlichkeit $F$ auf der y-Achse und dem Schadenausmass $E$ auf der $\mathrm{x}$-Achse mit einem Gradienten $g(B)$ 


$$
g(B)=\frac{\Delta(\log (F))}{\Delta(\log (E))}=-1
$$

Die Risikoaversion kann berücksichtigt werden, indem die Steigung der Akzeptanzlinie gemäss

$$
g(B) * \alpha=\mathrm{g}^{*}(\mathrm{~B})=\frac{\Delta\left(\log \left(F^{*}\right)\right)}{\Delta(\log (E))}<-1
$$

geändert wird: wenn sich das Schadenausmass $E$ verdoppelt, ist die akzeptable Eintretenswahrscheinlichkeit $F$ nicht mehr halb so gross, sondern weniger als halb so gross, so dass bei zunehmendem Schadenausmass die Eintretenswahrscheinlichkeit überproportional kleiner werden muss, um im akzeptablen Bereich zu bleiben. Da sie leicht verständlich ist, wird die Akzeptanzlinie oft in Verordnungen (z.B. Schweizerische Störfallverordnung, EIDGEnössisches DePARTEMENT DES INNERN 1989) verwendet.

Beim Aversions-Term wird die Risikoaversion $\alpha$ als variabler Faktor oder als konstanter Exponent in Abhängigkeit des Schadenausmasses $E$ berücksichtigt (SCHNEIDER 1996) und damit das wahrgenommene Risiko $R_{\text {perc }}$ berechnet:

$$
\begin{gathered}
R_{\text {perc }}=R_{o b j} \bullet \alpha(E)=F \bullet E \bullet \alpha(E) \\
R_{\text {perc }}=F \bullet E^{a}
\end{gathered}
$$

Die Festlegung des Aversionsausmasses wird im Rahmen eines Konsensfindungsprozesses der verantwortlichen staatlichen Stellen, Behörden und Experten vollzogen.

Jeweils gültig sind

$$
\begin{gathered}
\alpha(E)>1 \\
d \alpha / d E>0
\end{gathered}
$$

woraus folgt, dass Risiken mit einem grossen Schadenausmass künstlich progressiv vergrössert werden, während das Akzeptanzkriterium als konstant angenommen wird. Wird der Aversionsterm mit der Berechnung der Grenzkosten für die Schutzmassnahmen verwendet, kann abgeschätzt werden, welche Risiken akzeptabel sind und welche nicht (siehe BOHNENBLUST \& SCHNEIDER 1984).

Die Bezeichnung als wahrgenommenes Risiko $R_{\text {perc }}$ ist jedoch nicht korrekt, da neben dem Schadenausmass keine anderen Faktoren für die Bewertung des Risikos verwendet werden. Das resultierende Risiko wird besser als aversions-korrigiertes Risiko $R_{A C}$ bezeichnet.

\section{Das Ziel: Neues Modell der Risikobewertung}

Grundlegend gilt, dass das wahrgenommene Risiko $R_{\text {perc }}$ durch das objektive Risiko $R_{o b j}$ nicht beschrieben werden kann. Die Fokussierung auf das Schadenausmass gemäss der Gl. 2 reicht nicht aus, um Risiken korrekt zu bewerten. Die Berechnung des wahrgenommenen Risikos $R_{\text {perc }}$ muss auf relevanten Faktoren basieren, um eine möglichst exakte Berechnung des akzeptablen Risikos $R_{\text {acc }}$ zu ermöglichen. Gemäss Hollenstein (1997) sind dazu folgende Elemente nötig:

- Informationen über die Risiken

- Faktoren, welche die Verarbeitung der Information steuern

- Faktoren, welche die Information nutzbar machen

- Werthaltungen, mit denen Nutzen und Schaden definiert werden

- Präferenzen, um eine Zuordnung von Risiken und Werten vorzunehmen.

Es dürfte kaum möglich sein, all die aufgeführten Elemente zu berücksichtigen. Deshalb basiert der vorgestellte Ansatz auf folgenden Grundlagen:

- Informationen zum wahrgenommenen Risiko $R_{\text {perc }}$ : perception affecting factors PAF (siehe Tab. 1)

- Informationen zur Akzeptanz des Risikos $R_{a c c}$ : Evaluationskriterien $E C$ (siehe Tab. 1)

- personenbezogene Faktoren: «Modell-Evaluatoren» (siehe Kap. 5.4).

\section{Methoden}

In einem ersten Schritt (Abb. 2) wurden mittels Literaturrecherche die relevanten Informationen zur Risikowahrnehmung zusammengetragen. Parallel wurden die bestehenden Ansätze der Risikobewertung gruppiert und auf Stärken und Schwächen hin analysiert. Die Daten beider Schritte wurden anschliessend synoptisch zusammengetragen und Folgerungen für die Entwicklung des neuen Ansatzes abgeleitet.

Anschliessend wurde ein mathematisch formuliertes, theoretisches Modell der Risikobewertung entwikkelt. Dieses wurde einer Expertengruppe (Personen im Bereich Risikomanagement von Naturgefahren und/oder Risikowahrnehmung/-bewertung mit unterschiedlichen fachspezifischen Hintergründen) zur Prüfung vorgelegt (Qualitätssicherung). Die Relevanz der einzelnen Modellparameter für verschiedene Naturgefahrenprozesse wurde festgelegt und nun soll unter Beizug der prospect theory (KAHNEMAN \& Tversky 1979) die Quantifizierung vorgenommen werden. Zur Berücksichtigung der Menschen werden 


\begin{tabular}{|c|c|c|}
\hline$P A F / E C$ & Repräsentiert die Risiko-Charakteristika & Beschrieben u.a. von \\
\hline Freiwilligkeit & Freiwilligkeit & STARR (1969); FischHOF et al. (1978) \\
\hline Reduzierbarkeit & $\begin{array}{l}\text { Reduzierbarkeit } \\
\text { Vermeidbarkeit } \\
\text { Vorhersagbarkeit }\end{array}$ & $\begin{array}{l}\text { FischHof et al. (1978); SLovic et al. (1981); SLOVIC } \\
\text { et al. (1986); SLOVIC (1987) }\end{array}$ \\
\hline Furcht & $\begin{array}{l}\text { Kontrollierbarkeit } \\
\text { Anzahl der betroffenen Personen } \\
\text { Räumlich-zeitliche Verteilung der } \\
\text { Opfer } \\
\text { Grösse der betroffenen Fläche } \\
\text { Unmittelbarkeit der Auswirkungen } \\
\text { Direktheit der Auswirkungen }\end{array}$ & $\begin{array}{l}\text { FischHof et al. (1978); SLovic et al. (1981); SLOVIC } \\
\text { et al. (1986); SLovic (1987) }\end{array}$ \\
\hline Erfahrung & $\begin{array}{l}\text { Vertrautheit } \\
\text { Wissen über das Risiko } \\
\text { Handhabbarkeit }\end{array}$ & $\begin{array}{l}\text { FischHof et al. (1978); SLovic et al. (1981); SLOVIC } \\
\text { et al. (1986); SLovic (1987) }\end{array}$ \\
\hline
\end{tabular}

Tab. 1: Faktoren der Risikowahrnehmung und der Risikobewertung

Risk perception affecting factors and evaluation citeria

Facteurs de la perception du risque et de l'évaluation du risque

«Modell-Evaluatoren» entwickelt (siehe Kap. 5.4). Anschliessend erfolgt die Modellvalidierung mit Hilfe von Ergebnissen einer aktuellen Schweizer Studie zur Risikowahrnehmung (SIEGRIST et al. 2004) und den Resultaten einer Untersuchung in Deutschland (Plapp 2004).

\section{Theorie des neuen Ansatzes der Risikobewertung}

\subsection{Mathematische Formulierung}

Das wahrgenommene Risiko kann nicht durch das objektive Risiko definiert werden

$$
R_{o b j} \neq R_{p e r c}
$$

Durch den Beizug von wahrnehmungsbeeinflussenden Faktoren $P A F$ (Tab. 1) kann das wahrgenommene Risiko $R_{\text {perc }}$ berechnet werden

$$
R_{p e r c}=f\left(P A F, R_{o b j}\right)
$$

Angaben zur Risikoakzeptanz liefert die Risikobewertung

$$
R_{a c c}=f(E C)
$$

welche die Berechnung des akzeptablen Risiko $R_{\text {acc }}$ in Abhängigkeit des wahrgenommen Risikos $R_{\text {perc }}$ und der Evaluationskriterien $E C$ ermöglicht. Hypothetisch wird angenommen, dass die $E C$ den $P A F$ entsprechen.

Die Akzeptanz eines Risikos ist auch abhängig von individuellen, personenbezogenen Faktoren (siehe Kap. 5.4; Hollenstein 1997). Diese fliessen durch die Berücksichtigung von «Modell-Evaluatoren» in das Modell ein.

Die Entscheidung, ob ein Risiko aus der Sicht der Öffentlichkeit akzeptabel ist oder nicht, wird aufgrund des Vergleichs von $R_{\text {perc }}$ mit $R_{\text {acc }}$ getroffen:

$$
A c c_{i}=\left\{\begin{array}{l}
1 \text { wenn } R_{i, p e r c} \leq R_{i, a c c} \\
0 \text { wenn } R_{i, p e r c}>R_{i, a c c}
\end{array}\right.
$$

Dabei wird angenommen, dass $R_{i, a c c}$ konstant ist und die Beziehung

$$
A c c_{i}=f\left(P A F_{i}, R_{o b j, i}\right)
$$

gilt.

Das Modell der Risikobewertung basiert somit auf den Komponenten:

- Objektives Risiko $R_{o b j}$

- Faktoren der Risikowahrnehmung PAF

- Evaluationskriterien $E C$ 


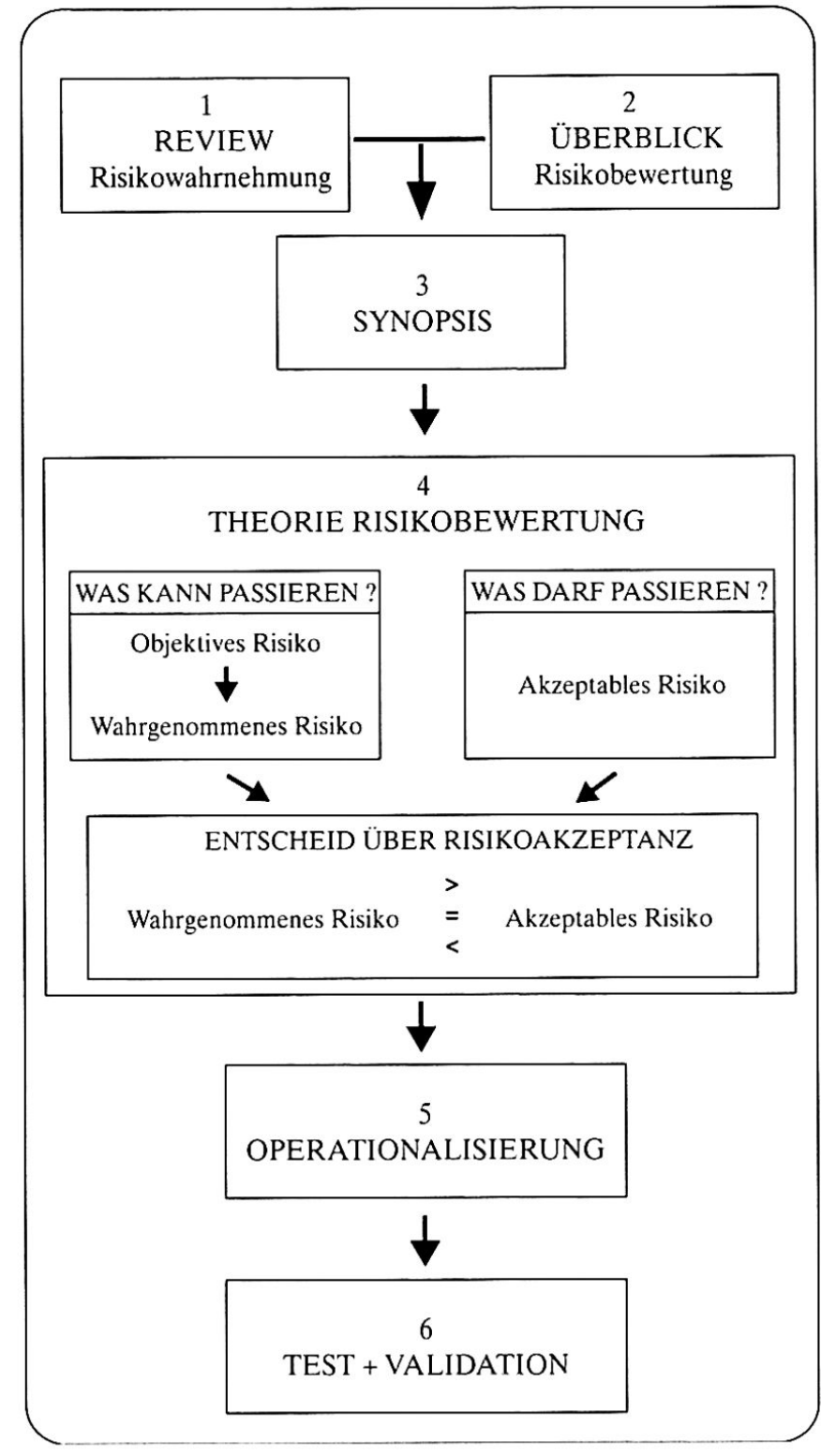

Abb. 2: Die sechs Vorgehensschritte

The multi-step procedure

Les six étapes

- Personenbezogene Faktoren (z.B. (Verhaltens-) Präferenzen und Werthaltungen der bewertenden Personen).

\subsection{Die Berïcksichtigung der Risikoaversion}

Die Risikoaversion wird nicht explizit als Funktion des Schadenausmasses berücksichtigt. Bei einem wörtlichen Verständnis des Begriffs Aversion ergibt die Verwendung der Modellparameter objektives Risiko $\mathrm{R}_{\text {obj }}$ (Schadenausmass $E$, Eintrittswahrscheinlichkeit $F$ ), wahrnehmungsrelevante Faktoren $P A F$ und Evaluationskriterien $E C$ jedoch eine indirekte Berücksichtigung der Risikoaversion. Alle Modellparameter besitzen, in
Abhängigkeit des untersuchten Naturgefahrenprozesses und der Region, in welcher der Prozess betrachtet wird, eine aversive, und eine non-aversive Komponente (Abb. 3).

Eine Lawine im Gebirge kann ein grosses Schadenausmass bewirken, hat zudem eine grosse Eintretenswahrscheinlichkeit, wird meist unfreiwillig in Kauf genommen und hat insgesamt ein grosses Bedrohungspotential (Schrecklichkeit). Dies vergrössert die Abneigung gegen das Risiko einer Lawine. Andererseits können die gute Reduzierbarkeit der Lawinengefahr sowie die grosse Erfahrung und das Wissen, das im Umgang mit Lawinen vorhanden ist, dazu führen, dass die Abneigung gegen dieses Risiko geringer wird. Diese Informationen müssen für die Quantifizierung der aversiven oder nicht-aversiven Wirkung verwendet werden (siehe exemplarisch Abb. 3). Die Verknüpfung der Wirkungen der einzelnen Modellparameter ergibt die Gesamtwirkung.

\subsection{Ungleiche Gewichtung von Gewinnen und Verlusten}

Entscheidungstheoretische Erkenntnisse (siehe dazu z.B. SIMON 1996) führen zu einer besseren Abbildung des menschlichen Verhaltens unter Unsicherheit. Rationale Faktoren des Risikos (Eintretenswahrscheinlichkeit, Schadenausmass) spielen für die Risikowahrnehmung und -bewertung oft nur eine untergeordnete Rolle. Gemäss der utility theory (SIMON 1996) hat der homo oeconomicus vollständige Informationen über alle relevanten Aspekte einer Entscheidung und zudem ein stabiles, gut strukturiertes Präferenzsystem. Somit ist er fähig,seine Entscheidung mittels einer Präferenzskala zu bewerten und die Handlungsalternativen auf dieser Bewertungsskala einzuordnen. Dadurch fällt er den Entscheid zugunsten oder zuungunsten einer Handlungsalternative aufgrund rationaler Überlegungen, die er sich zum endgültigen Zustand als Folge einer solchen Alternative macht. Bei der bounded rationality (SIMON 1996) führen fragmentarische und fehlerhafte Informationen über die relevanten Aspekte einer Entscheidung (= eingeschränkte Rationalität) zu einer realistischeren Abbildung des «irrationalen» menschlichen Verhaltens. Die beste Abbildung des «irrationalen» Verhaltens unter Unsicherheit ermöglicht jeodch die prospect theory (KAHNEMAN \& TVERSKY 1979), die von unvollständigen Informationen über die relevanten Aspekte einer Entscheidung ausgeht. Sie basiert auf der Annahme, dass bei Entscheiden nicht der mögliche Endzustand einer Handlungsalternative, sondern der mögliche Betrag des Gewinns oder des Verlustes (in Abb. 4 auf der Achse $x$ eingezeichnet), der durch die Wahl einer Handlungsalternative erwartet werden kann, relevant ist. Der Wert dieses Gewinns oder Verlustes (in Abb. 4 auf der Achse $v(x)$ eingezeichnet) wird mit Hilfe eines Referenzpunktes $v(x)=0$, der 


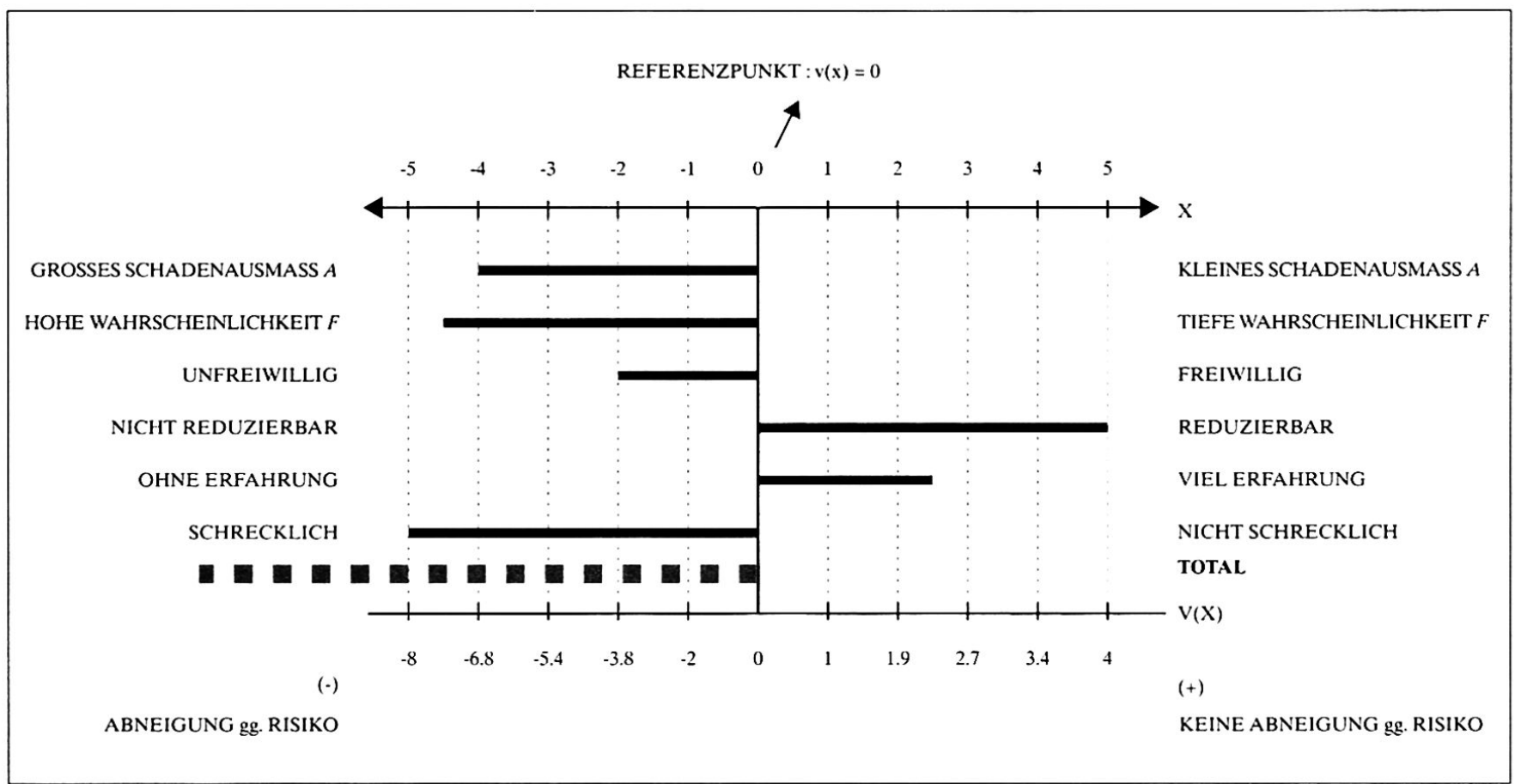

Abb. 3: Quantifizierung der Modellparameter

Da die Modellparameter nicht für alle Naturgefahren gleich relevant sind und negative Folgen («Verluste») stärker zu gewichten sind als positive Folgen ( «Gewinne»; siehe Abb. 4), bieten sich z.B. nicht-lineare Werte für die Gewichtung an. Im dargestellten Beispiel bezeichnet dabei der Wert $\mathrm{v}(\mathrm{x})=3.8$ die Gewichtung, die z.B. der Wert $\mathrm{x}=2$ erhält. (Zahlen und Werte sind exemplarisch zu verstehen.)

Quantification of the model parameters

Quantification des paramètres de modélisation

die Rolle des Ausgangswerts übernimmt, berechnet. Ein zahlenmässig gleich hoher Verlust wird gegenüber einem Gewinn stärker gewichtet (Verlustaversion).

Somit kann z.B. der Entscheid, ob Lawinenschutzmassnahmen getroffen werden, einen Einfluss auf den Bodenpreis der im Einzugsbereich des Lawinenzugs gelegenen Flächen haben. Denn, ausgehend vom gegenwärtigen Wert des Bodens, kann festgestellt werden, dass bei einem Treffen der Schutzmassnahmen der Bodenwert zunimmt, z.B. um 2'000.- Sfr., während bei einem Verzicht ein Wertverlust von 2'000.- Sfr. konstatiert werden muss (siehe Abb. 4). Gemäss der prospect theory wird dieser Verlust stärker gewichtet als der nominell gleich hohe Gewinn (vergleiche Punkt $V$ mit Punkt $G$ in Abb. 4). Folgerichtig werden Verluste, die infolge eines Entscheides (in diesem Fall, ob die Lawinenschutzmassnahmen getroffen werden oder nicht) auftreten können, als grösser wahrgenommen als die nominell gleich hohen Gewinne.

Die Berücksichtigung dieser Erkenntnis bei der Quantifizierung der Modellparameter könnte z.B. wie folgt geschehen (siehe Abb. 3 und Abb. 4): Auf der Achse $v(x)$ (Werte sind exemplarisch zu verstehen) sind nicht- lineare Werte zu finden, mit denen das Gewicht einer Grösse $x$ angegeben wird. Für das angeführte Beispiel der Lawine im Gebirge bedeutet dies, dass der Schadenausmasswert $x=-4$ ein Gewicht von $v(x)=-6.8$ hat. Gemäss diesem Vorgehen können auch die anderen Parameter des Modells gewichtet und quantifiziert werden. Somit kann der Einfluss der Parameter auf die Abneigung gegenüber einem Risiko, und letztendlich dessen Akzeptanz, definiert werden.

5.4 Die wahrnehmende Person: «Modell-Evaluatoren» Die Bewertung eines Risikos durch eine Person wird durch eine Vielzahl von Faktoren beeinflusst (siehe Tab. 2).

Basierend auf der Wahrscheinlichkeitsverteilung dieser Faktoren in der Bevölkerung (z.B. gemäss statistischen Daten) und einer Monte-Carlo-Simulation können «Modell-Evaluatoren» generiert werden. Diese ermöglichen die zusammenfassende Charakterisierung einer grossen Anzahl verschiedener Menschentypen. Die «Modell-Evaluatoren» sind im Modell die Stellvertreter der Menschen und haben eine eigene, individuelle Risikowahrnehmung und -bewertung. Basierend 


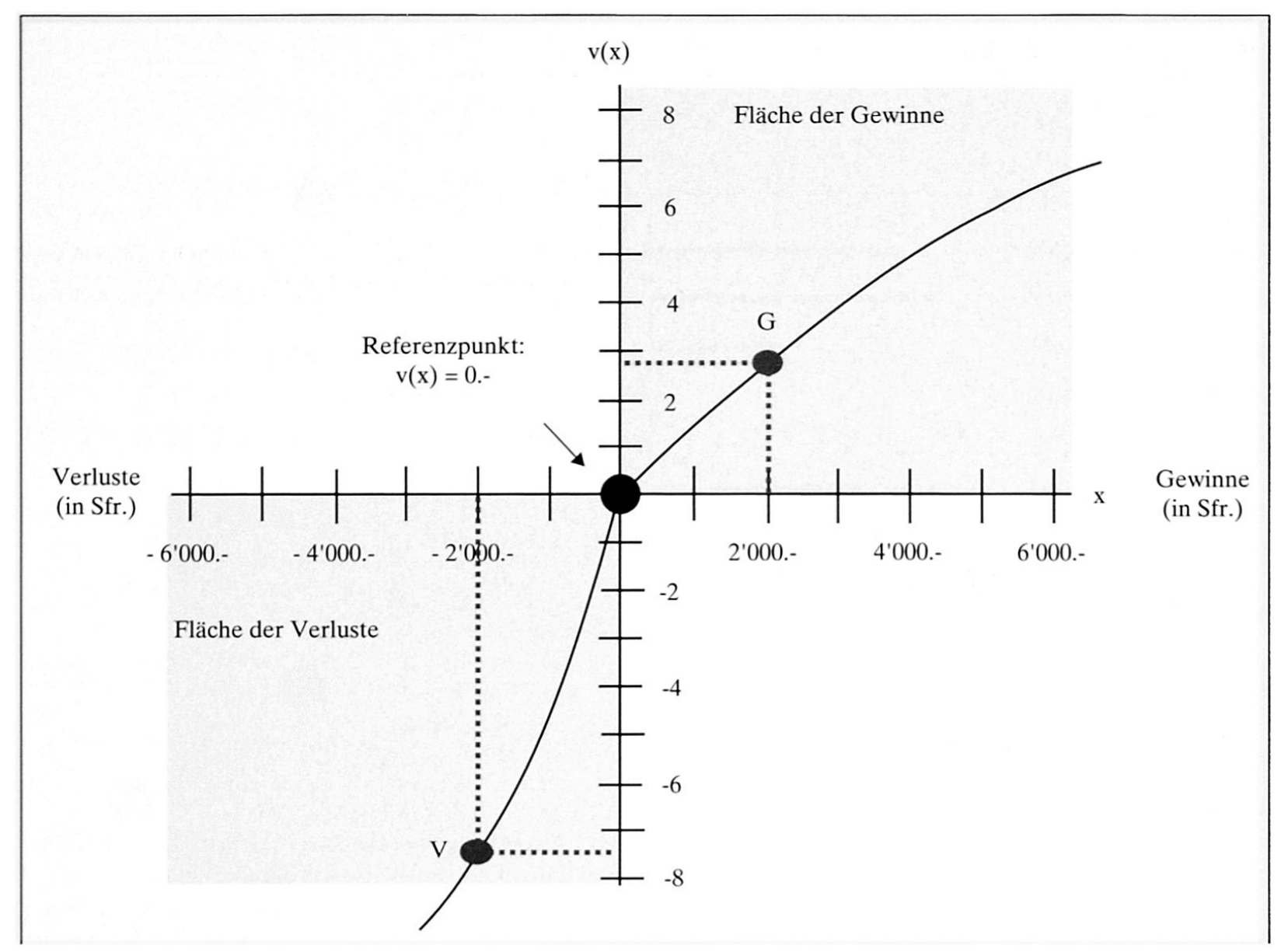

Abb. 4: Verluste werden stärker gewichtet als Gewinne.

Infolge Risikoreduktionsmassnahmen können höhere Bodenpreise erzielt werden (z.B. Punkt G: +2’000.- Sfr.). Werden die Schutzmassnahmen nicht ergriffen, fallen die Preise (z.B. Punkt V: -2’000.- Sfr.). An der Achse v(x) ist ersichtlich, dass Verluste stärker gewichtet werden. (Zahlen und Werte sind exemplarisch zu verstehen.)

Losses are stronger weighted than gains.

Les pertes ont un poids plus important que les gains.

auf der Gl. 14 kann daher das kollektiv wahrgenommene und akzeptable Risiko berechnet und gemäss Gl. 12 der Entscheid über die Akzeptanz des Risikos gefällt werden

$$
R_{\text {coll }}=\sum_{i=1}^{n} r_{\text {ind.i }}
$$

\section{Diskussion}

\subsection{Verwendung der Risikoaversion}

Bei den gängigen Bewertungsansätzen ist das Ausmass der Risikoaversion das Ergebnis eines Konsensfindungsprozesses innerhalb der verantwortlichen Institutionen und Behörden. Das Ausmass der Aversion ist somit keine objektive Grösse. Die Wahrnehmung von Risiken ist ein subjektiver und intuitiver Prozess, so dass das Problem der fehlenden Objektivität kaum gelöst, sondern allenfalls minimiert werden kann. Bei der Berücksichtigung der öffentlichen kollektiven Risikowahrnehmung können, trotz Vereinfachungen, nur annähernd korrekte Resultate erreicht werden. Der vorgestellte Ansatz berücksichtigt die Aversion daher nicht mehr explizit, sondern indirekt durch die Gewichtung der $P A F$ und $E C$.

\subsection{Faktoren der Risikowahrnehmung und -bewertung}

Die Auswahl der $P A F$ und der $E C$ stellt nur einen kleinen Ausschnitt der risikobezogenen relevanten Faktoren der Risikobewertung dar. Da aber nicht das perfekte, allumfassende Modell angestrebt wird, sondern eine Verbesserung gegenüber den gängigen Ansätzen, ist die Fokussierung auf wenige Faktoren vertretbar. Der Versuch, die qualitativen, nicht-normativen Fak- 


\begin{tabular}{|ll|}
\hline Personenbezogene Faktoren & Beispiele \\
\hline Ökonomische Perspektiven & Arm versus vermögend \\
Soziale Umwelt & Strukturelle und kulturelle Eigenschaften der sozialen Gruppe \\
Werte und Weltbilder & $\begin{array}{l}\text { z.B. technokratisch versus naturalistisch, progressiv versus } \\
\text { konservativ, individualistisch versus kollektivistisch }\end{array}$ \\
$\begin{array}{l}\text { Psychologische und } \\
\text { Verhaltenscharakteristika } \\
\text { (Präferenzen) }\end{array}$ & z.B. risikofreudig versus risikoavers \\
\hline
\end{tabular}

Tab. 2: Personenbezogene Faktoren der Risikowahrnehmung und -bewertung Person-related factors of risk perception and risk evaluation Facteurs de perception et d'évaluation du risque se rapportant aux personnes

toren $P A F$ und $E C$ in normative Grössen zu wandeln (Skalierung und Quantifizierung) stellt jedoch eine berechtigte Quelle der Kritik dar.

Auch bei den personenbezogenen Faktoren gibt es viele, die für die Risikobewertung eines Individuums wichtig sind. Nicht alle können in einem Modell berücksichtigt werden. Eine Vereinfachung ist hier nötig. «Modell-Evaluatoren» ermöglichen dabei, die Wirkung der Faktoren auf einer kollektiven Ebene zu berücksichtigen. Solch ein Vorgehen ist nicht optimal und wird der Vielschichtigkeit der Menschen in keiner Weise gerecht. Doch aus Gründen der Praktikabilität kann es gut begründet werden.

\section{Fazit}

Beim Umgang mit Naturgefahren müssen die gesellschaftlichen Anliegen und Bedürfnisse berücksichtigt werden. Ein risiko-orientierter Ansatz bedingt die Integration der Öffentlichkeit. Daher muss die öffentliche Akzeptanz von Naturrisiken eruiert werden, so dass Unterschiede zwischen den Einschätzungen der verantwortlichen Stellen und der Öffentlichkeit erkennbar werden. Die Simulation der öffentliche Risikobewertung stellt hier eine attraktive Option dar, weil sie kosten- und zeitintensive Bevölkerungsbefragungen ersetzen kann. Trotz dieser Vorteile kann sie auch kritisiert werden, denn ein Modell kann die öffentliche Risikobewertung nie fehlerfrei abbilden. Daher darf dieses nicht anstelle einer vertieften Diskussion der verantwortlichen Stellen mit der Bevölkerung verwendet werden. Seinen Zweck erfüllt es als Mittel, um beim Risikomanagement bessere Lösungen zu finden. Es darf aber nicht das einzige Entscheidungs- kriterium sein. Vergleiche mit anderen Risiken oder behördlichen Richtlinien sind genauso wichtig, um sinnvolle Lösungen zu erhalten.

\section{Literatur}

BOHNEnBlust, H. \& T. SCHNEIDER (1984): Risk appraisal - can it be improved by formal decision models? In: Covello, V.T., LaVe, L.B., Moghissi, A. \& V.R.R. UPPULURI (Hrsg.): Uncertainty in risk assessment, risk management and decision making. - = Advances in risk analysis 4, New York, London: 71-87.

Bohnenblust, H. \& C. Troxler (1987): Risk analysis - is it a useful tool for the politician in making decisions on avalanche safety? - In: SALM, B. \& H. GUbleR (Hrsg.): Davos Symposium: avalanche formation, movement and effects. - = International association of hydrological sciences (IAHS) 162, Davos: 653-664.

BUNDESAMT FÜr UMWELT, WALD UND LANDSCHAFt (BUWAL) (1999): Kosten-Wirksamkeit von Lawinenschutz-Massnahmen an Verkehrsachsen. $-=$ Vollzug Umwelt, Bern:1-110.

Bundesamt Für Zivilschutz (BZS) \& ERnst Basler + Partner AG (1995): Katastrophen und Notlagen in der Schweiz (KATANOS). Eine vergleichende Übersicht. - Bern: Bundesamt für Zivilschutz.

Douglas, M. \& A. Wildavsky (1982): Risk and culture. - Berkeley, Los Angeles, London: University of California Press.

EIDGENössisches Departement des InNeRn (EDI) (1989): Verordnung über den Schutz vor Störfällen (Störfallverordnung). - Bern.

FARMER, F.R. (1967): Siting criteria - a new approach. - In: International Atomic Energy Agency (IAEA) (Hrsg.): Containment and siting of nuclear power plants. - Wien: 303-329. 
Fischioff, B., Slovic, P., Lichtenstein, S., Read, S. \& B. Combs (1978): How safe is safe enough ? A psychometric study of attitudes towards technological risks and benefits. - In: Policy Sciences 9, 2: 127-152.

HeinimanN, H.R. (2002): Risk management - a framework to improve effrectiveness and efficiency of resource management decisions. - In: GREMINGER, P. (Hrsg.): $23^{\text {rd }}$ session of the European Forestry Commission's working party on the management of mountain watersheds. - Bern: Bundesamt für Umwelt, Wald und Landschaft (BUWAL): 16-19.

Hollenstein, K. (1997): Analyse, Bewertung und Management von Naturrisiken: - Zürich: vdf Hochschulverlag ETH Zürich.

Kahneman, D. \& A. Tversky (1979): Prospect Theory: an analysis of decision under risk. - In: Econometrica 47, 2: 263-291.

Kasperson, R.E., Renn, O., Brown, H.S., Emel, J., Goble, R., Kasperson, J.X. \& S. Ratick (1988): The social amplification of risk: a conceptual framework. In: Risk Analysis 8, 2: 177-187.

PlaPP, T. (2004):Wahrnehmung von Risiken aus Naturkatastrophen. Eine empirische Untersuchung in sechs gefährdeten Gebieten Süd- und Westdeutschlands. = Karlsruher Reihe II, Risikoforschung und Versicherungsmanagement 2, Karlsruhe: Verlag für Versicherungswirtschaft.

SCHNEIDER, J. (1996): Sicherheit und Zuverlässigkeit im Bauwesen. Grundwissen für Ingenieure. - Zürich: vdf Hochschulverlag ETH Zürich.

SCHNEIDER, T. (1985): Ein quantitatives Entscheidungsmodell für Sicherheitsprobleme im nicht-nuklearen Bereich. - In: Yadigaroglu, Y. \& S. Chakraborty (Hrsg.): Risikountersuchungen als Entscheidungsinstrument - Risk analysis as a decision tool. - Köln: Verlag TÜV Rheinland: 113-143.

Siegrist, M., Gutscher, H., Orlow, P. \& Ü. Yoker (2004): Hochwassergefahren in der Schweiz: Risikobewusstsein in der Bevölkerung und die Implikationen für eine erfolgreiche Risikokommunikation. - Unveröffentlichter Schlussbericht, Studie im Auftrag der Nationalen Plattform Naturgefahren (PLANAT), bearbeitet durch das Institut für Sozialpsychologie der Universität Zürich, Sozialforschungsstelle.

Simon, H.A. (1996): The science of the artificial. Boston: MIT Press.

Slovic, P., Fischroff, B. \& S. Lichtenstein (1981): Perceived risk: psychological factors and social implications. - In: Proceedings of the Royal Society of London. Series A, Mathematical and Physical Sciences 376, 1764: 17-34.

Slovic, P., Fischioff, B. \& S. Lichtenstein (1986): The psychometric study of risk perception. - In: Covello, V.T., Menkes, J. \& J. Mumpower (Hrsg.): Risk evaluating and management. $-=$ Contemporary issues in risk analysis, New York: 3-24.
SLovic, P. (1987): Perception of risk. - In: Science. New Series 236, 4799: 280-285.

SOCIETY OF RISK ANALYSIS (SRA) (2004): Glossary of risk analysis. - http://www.sra.org/resources_glossary.php 22.04.2004.

Starr, C. (1969): Social benefit versus technological risk. - In: Science. New Series 165, 3899: 1232-1238.

TVersky, A. \& D. Kahneman (1974): Judgement under uncertainty: heuristics and biases. - In: Science. New Series 185, 4157: 1124-1131.

WILDAVSKY, A. \& K. DAKE (1990): Theories of risk perception: who fears what and why? - In: Daedalus 119, 4: 41-60.

\section{Zusammenfassung: Die Bewertung von Risiken durch Naturgefahren mit spezieller Berücksichtigung der Risikoaversion}

Ein Vergleich des effektiven Risikos $R_{o b j}$ (Risikoanalyse) mit dem akzeptablen Risiko $R_{a c c}$ (Risikobewertung) ermöglicht im Risikomanagement die Planung notwendiger Massnahmen, die zu hohe objektive Risiken auf ein akzeptables Mass reduzieren oder deren Anstieg darüber hinaus verhindern sollen. In der formalen Risikobewertung wird die Risikoaversion $\alpha$, die als Funktion des Schadenausmasses $E$ eines Ereignisses zu verstehen ist $(\alpha=f(E)=\alpha(E))$, als Stellvertreter für die öffentliche Wahrnehmung verwendet. Der hier vorgestellte Ansatz geht davon aus, dass die Risikoaversion $\alpha$ nicht ausreicht, um diese Wahrnehmung zu modellieren. Die relevanten Faktoren $(P A F, E C)$ werden in einem mathematischen Modell integriert und können eine aversive oder nicht-aversive Komponente besitzen. Dieser Ansatz soll zur Unterstützung der Entscheidungsfindung im Rahmen des Risikomanagements verwendet werden können.

\section{Summary: Evaluation of Natural Hazard Risks with Special Consideration of Risk Aversion}

A comparison of the effective risk $R_{o b j}$ (risk analysis) with the permissable risk $R_{\text {acc }}$ (risk evaluation) allows for integral action planning within risk management, aiming at a reduction of unacceptable risks to an acceptable level and maintaining acceptable risk at that level. Common formal evaluation approaches use the concept of risk aversion $\alpha$, defined as a function of the extent of damage $E: \alpha=f(E)=\alpha(E)$, as a surrogate for public risk perception. The proposed approach is based on the assumption that risk aversion $\alpha$ alone does not suffice for a simulation of risk perception. The perception affecting factors $(P A F)$ and evaluation criteria $(E C)$ are integrated in a mathematically formulated model and possesses both an aversive and a non-aversive component. It should thereby be possible to simulate public risk evaluation and to use the model as a tool in the risk management process. 
Résumé: L'évaluation des risques inhérents aux dangers naturels, par la prise en considération spécifique de l'aversion pour le risque

La comparaison du risque effectif $R_{o b j}$ (analyse du risque) avec le risque acceptable $R_{\text {acc }}$ (évaluation du risque) permet d'améliorer les prévisions en vue d'une réduction de risques de trop grande portée à un niveau acceptable, ou d'empêcher la progression de ces risques. Dans l'appréciation formelle du risque, l'aversion pour le risque $\alpha$, qui doit être appréhendée comme une fonction de l'importance des dégâts $E$ occasionnés par un événement $(\alpha=f(E)=\alpha(E))$, est considérée comme l'expression de la perception publique. L'approche, qui est exposée ici, part du principe que l'aversion pour le risque $\alpha$ est insuffisante pour permettre la modélisation de cette perception. Les facteurs déterminants $(P A F, E C)$ sont intégrés à un modèle mathématique et peuvent comporter une composante aversive et nonaversive. Cette approche est destinée à renforcer le rôle de la découverte décisionnelle dans la gestion du risque.

Dipl.-Geogr. Thomas Plattner, Professur für Forstliches Ingenieurwesen, ETH Zürich, Rämistrasse 101, CH-8092 Zürich.

e-mail: thomas.plattner@env.ethz.ch

Manuskripteingang/received/manuscrit entré le 26.4.2004

Annahme zum Druck/accepted for publication/accepté pour l'impression: 8.3.2005 